\title{
SINGULAR INTEGRAL EQUATIONS OF THE VOLTERRA TYPE*
}

BY

\author{
CLYDE E. LOVE
}

1. Introduction. It has been shown by Evans $\dagger$ that the equation

$$
\phi(x)=f(x)+\int_{x}^{\infty} K(x, t) \phi(t) d t
$$

has a unique finite and integrable solution provided that, for all values of $x \geqq a$ and of $t \geqq x,(a)$ the functions $f(x)$ and $K(x, t)$ are bounded and continuous; (b) the integral

$$
\int_{x}^{\infty}|K(x, t)| d t
$$

exists; $(c)$ a constant $b$ can be found such that

$$
\int_{x}^{\infty}|K(x, t)| d t \leqq N<1 \quad(x \geqq b) .
$$

Equations of the form (1) sometimes arise, $\ddagger$ however, for which the conditions of Evans's theorem are not satisfied. Various cases in which this is true are considered in the present paper. In each instance an attempt is made not merely to prove the existence of a continuous solution, but also to determine its behavior for large values of $x$.

The independent variables $x$ and $t$ will be restricted to real values. Functions of the single variable $x$ will be considered for values of $x$ lying either on the range

$I$

or on the range

$$
I_{b}
$$

$$
x \geqq a
$$$$
a \leqq x \leqq b,
$$

* Presented to the Society, September 8, 1914.

†Atti della Reale Accademia dei Lincei, ser. 5, vol. 20 (1911), pp. 656-662. Cf. also: Ibid., vol. 20 (1911), pp. 7-11.

$\ddagger$ For instance, in the theory of linear differential equations. Cf. Dini, Annali di M a t e m a t i c a , ser. 3, vol. 2 (1899), pp. 297-324; ibid., vol. 3 (1900), pp. 125-183; also papers by the present writer: Annals of Mathematics, ser. 2, vol. 15 (1914), pp. 145-156, and American Journal of Mathematics, vol. 36 (1914), pp. $151-166$. 
where $b$ is arbitrary; functions of the two variables $x, t$ will be considered for values of $x$ and $t$ in the domain

$$
x \geqq a, \quad t \geqq x .
$$

For brevity we shall speak of these three regions as the interval $I$, the interval $I_{b}$, and the region $T$ respectively.

The functions $f(x)$ and $K(x, t)$ are assumed throughout to be continuous in $I$ and in $T$ respectively.

2. Theorem 1. If the integral

$$
\int_{x}^{\infty}|K(x, t) f(t)| d t
$$

converges in $I$, converges uniformly in $I_{b}$, and has in $I$ the property that

$$
\int_{x}^{\infty}|K(x, t) f(t)| d t<\theta|f(x)| \quad(0<\theta<1),
$$

where $\theta$ is a constant independent of $x$, then equation (1) has in I a continuous solution $\phi(x)$ expressible in the form

$$
\phi(x)=f(x) \rho(x),
$$

where $\rho(x)$ is a continuous function such that

$$
|\rho(x)|<1 /(1-\theta) .
$$

The method of iteration, ${ }^{*}$ when applied to the equation (1), leads to consideration of the series

$$
\phi(x)=\sum_{n=0}^{\infty} u_{n}(x)
$$

where

$$
\begin{gathered}
u_{0}(x)=f(x), \\
u_{n}(x)=\int_{x}^{\infty} \int_{t}^{\infty} \cdots \int_{t_{n-3}}^{\infty} \int_{t_{n-2}}^{\infty} K(x, t) K\left(t, t_{1}\right) \\
\cdots K\left(t_{n-3}, t_{n-2}\right) K\left(t_{n-2}, t_{n-1}\right) f\left(t_{n-1}\right) d t_{n-1} d t_{n-2} \cdots d t_{1} d t \\
=\int_{x}^{\infty} K(x, t) u_{n-1}(t) d t \quad(n=1,2, \cdots) .
\end{gathered}
$$

We shall first show by mathematical induction that, in $I, u_{n}(x)$ is continuous and satisfies the inequality

$$
\left|u_{n}(x)\right|<\theta^{n}|f(x)| \quad(n=1,2, \cdots) .
$$

* Cf., for example, Bôcher, An Introduction to the Study of Integral Equations (1909), p. 14. 
Assume that $u_{n-1}(x)$ is continuous in $I$, and that

$$
\left|u_{n-1}(x)\right| \leqq \theta^{n-1}|f(x)| \text {. }
$$

Then $u_{n}(x)$ is continuous in $I$ and satisfies (5). For, since the integral

$$
\int_{x}^{\infty}|K(x, t) f(t)| d t
$$

converges uniformly in $I_{b}$, we can find for every value of $\epsilon>0$ a value of $\mu$ independent of $x$, such that

$$
\int_{\mu}^{u^{\prime}}|K(x, t) f(t)| d t<\epsilon,
$$

where $\mu^{\prime}$ has any value greater than $\mu$, and $x$ lies in $I_{b}$. Now

$$
\begin{aligned}
\left|\int_{\mu}^{\mu^{\prime}} K(x, t) u_{n-1}(t) d t\right| \leqq \int_{\mu}^{\mu^{\prime}} \mid K(x, t) & u_{n-1}(t) \mid d t \\
& \leqq \theta^{n-1} \int_{\mu}^{\mu^{\prime}}|K(x, t) f(t)| d t<\epsilon,
\end{aligned}
$$

by (5) and (6). But this last relation establishes the uniform convergence of $u_{n}(x)$ in $I_{b}$, and hence its continuity at all points in $I$, since an interval $I_{b}$ can always be found so as to include any preassigned point in $I$. Further,

$$
\left|u_{n}(x)\right| \leqq \int_{x}^{\infty}\left|K(x, t) u_{n-1}(t)\right| d t \leqq \theta^{n-1} \int_{x}^{\infty}|K(x, t) f(t)| d t<\theta^{n}|f(x)|,
$$

To complete the proof by induction we need only note that our assumption regarding $u_{n-1}(x)$ is justified, by hypothesis, for $n=1$.

As a consequence of (5), we have

$$
\sum_{n=0}^{1 \infty}\left|u_{n}(x)\right|<\frac{1}{1-\theta}|f(x)|,
$$

so that the series $\sum_{n=0}^{n=\infty} u_{n}(x)$ is absolutely convergent in $I$, and $\phi(x)$ as given by (4) is defined in that interval. Further, the same series is uniformly convergent in $I_{b}$, by Weierstrass's test, since

$$
\left|u_{n}(x)\right|<N \theta^{n},
$$

where $N$ is the maximum value of $|f(x)|$ in $I_{b}$. The function $\phi(x)$ is therefore the sum of a uniformly convergent series of continuous functions, and is itself continuous.

Let us now multiply the series for $\phi(t)$ by $K(x, t)$ and integrate term by term from $x$ to $\infty$. That this is allowable appears from the following theorem:*

* Bromwich, Infinite Series (1908), p. 453. 
If the series $\sum_{n=0}^{n=\infty} f_{n}(x)$ converges uniformly in any fixed interval $a \leqq x \leqq b$, where $b$ is arbitrary, and if $F(x)$ is continuous for all finite values of $x$, then

$$
\int_{a}^{\infty} F(x)\left[\sum_{n=0}^{\infty} f_{n}(x)\right] d x=\sum_{n=0}^{\infty} \int_{a}^{\infty} F(x) f_{n}(x) d x,
$$

provided that the integral.

converges.

$$
\Phi(x)=\int_{a}^{\infty}|F(x)| \sum_{n=0}^{\infty}\left|f_{n}(x)\right| d x,
$$

For, if we take $a=x, x=t, F(t)=K(x, t), f_{n}(t)=u_{n}(t)$, it is only necessary to show that the integral $\Phi(x)$ converges. But this follows at once from (7) and (2).

Upon performing the term-by-term integration mentioned above, we obtain the equation

$$
\int_{x}^{\infty} K(x, t) \phi(t) d t=\sum_{n=1}^{\infty} u_{n}(x)=\phi(x)-f(x),
$$

which shows that $\phi(x)$ as given by (4) is a solution of (1).

From (7) it appears at once that we may write $\phi(x)$ in the form (3). The function $f(x)$ cannot vanish in $I$, by (2), so that $\rho(x)$ is continuous. This completes the proof of the theorem.

That the solution thus obtained is not necessarily the only continuous solution* appears from the following example. The hypotheses of the theorem are evidently satisfied by the equation

since

$$
\phi(x)=e^{x}+\frac{1}{2} e^{2 x} \int_{x}^{\infty} e^{-2 t} \phi(t) d t
$$

$$
\int_{x}^{\infty}|K(x, t) f(t)| d t=\frac{1}{2} e^{2 x} \int_{x}^{\infty} e^{-t} d t=\frac{1}{2} e^{x}
$$

The equation evidently has the infinity of solutions

$$
\phi(x)=2 e^{x}+c e^{3 x / 2},
$$

where $c$ is an arbitrary constant, the solution given by the theorem being obtained by taking $c=0$.

Similar examples can be constructed for each of the cases still to be treated. In case the hypotheses of the theorem are not satisfied for all values of

* It may be remarked that in the case treated by Evans (loc. cit.) there may likewise be more than one continuous solution. The equation

$$
\phi(x)=1+\frac{1}{2} e^{x} \int_{x}^{\infty} e^{-t} \phi(t) d t,
$$

which evidently satisfies the conditions of Evans's theorem, has the family of continuous solutions

where $c$ is an arbitrary constant.

$$
\phi(x)=2+c e^{x / 2},
$$


$x$ in $I$, but only for sufficiently large values, say $x \geqq a^{\prime}>a$, we may proceed by "prolongement"* to obtain a continuous solution valid throughout $I$. Write the equation (1) in the form

$$
\phi(x)=f_{1}(x)+\int_{x}^{a^{\prime}} K(x, t) \phi(t) d t
$$

where

$$
f_{1}(x)=f(x)+\int_{a^{\prime}}^{\infty} K(x, t) \phi(t) d t .
$$

Our theorem may be used to determine $\phi(x)$ for $x \geqq a^{\prime}$, after which $f_{1}(x)$ becomes a known function, and (8) reduces to a regular Volterra equation whose solution in the interval $a \leqq x \leqq a^{\prime}$ may be found by the usual methods.

A similar remark will apply to each of the later theorems.

3. Theorem 2. If there exists a continuous positive real function $h(x)$ such that the integrals

$$
\int_{x}^{\infty}|K(x, t) f(t)| d t, \quad \int_{x}^{\infty}|K(x, t)| h(t) d t
$$

converge in $I$, converge uniformly in $I_{b}$, and satisfy in $I$ the inequalities

$$
\begin{aligned}
& \int_{x}^{\infty}|K(x, t) f(t)| d t<h(x), \\
& \int_{x}^{\infty}|K(x, t)| h(t) d t<\theta h(x) \quad(0<\theta<1),
\end{aligned}
$$

where $\theta$ is a constant independent of $x$, then equation (1) has in I a continuous solution $\phi(x)$ expressible in the form

$$
\phi(x)=f(x)+h(x) \rho(x),
$$

where $\rho(x)$ is a continuous function such that

$$
|\rho(x)|<1 /(1-\theta) \text {. }
$$

Corollary. If the integrals

$$
\int_{x}^{\infty}|K(x, t) f(t)| d t, \quad \int_{x}^{\infty}|K(x, t)| d t
$$

converge in $I$, converge uniformly in $I_{b}$, and satisfy in $I$ the inequalities

$$
\int_{x}^{\infty}|K(x, t) f(t)| d t<N, \quad \int_{x}^{\infty}|K(x, t)| d t<\theta,
$$

where $N$ is a constant, then equation (1) has in I a continuous solution $\phi(x)$ expressible in the form

where

$$
\begin{gathered}
\phi(x)=f(x)+\rho(x), \\
|\rho(x)|<N /(1-\theta) .
\end{gathered}
$$

\footnotetext{
* Cf. Evans, loc. cit., p. 662.
} 
To prove the theorem, consider again the series (4). We note first that $u_{n}(x)$ is continuous in $I$ and satisfies the inequality

$$
\left|u_{n}(x)\right|<\theta^{n-1} h(x) \quad(n=1,2, \cdots) .
$$

For, if we assume that $u_{n-1}(x)$ has these properties, the same will be true for $u_{n}(x)$, by argument similar to that of $\S 2$. But the assumption holds for $u_{1}(x)$, by hypothesis.

It follows from (12) that the series (4) converges absolutely in $I$ and uniformly in $I_{b}$. By making obvious modifications in the argument of $\S 2$, we see that $\phi(x)$ as given by (4) is continuous in $I$, and satisfies equation (1) in the same interval.

By (12), we have

so that

$$
\sum_{n=1}^{\infty}\left|u_{n}(x)\right|<\frac{1}{1-\theta} h(x),
$$

$$
|\phi(x)-f(x)|<\frac{1}{1-\theta} h(x) .
$$

The form (11) for $\phi(x)$ results at once.

The corollary is merely that case of the theorem in which we may take

$$
h(x)=N .
$$

4. Theorem 3. If there exists a continuous positive real function $\eta(x)$ for which the integral

exists, and such that, in $T$,

$$
\int_{x}^{\infty} \eta(t) d t
$$

$$
|K(x, t) f(t)|<|f(x)| \eta(t),
$$

then equation (1) has in I a continuous solution $\phi(x)$ expressible in the form

$$
\phi(x)=f(x)[1+\epsilon(x)],
$$

where $\epsilon(x)$ is continuous and has the property that

$$
\lim _{x=\infty} \epsilon(x)=0 .
$$

We may evidently assume without loss of generality that

$$
\int_{x}^{\infty} \eta(t) d t<\theta \quad(0<\theta<1) .
$$

For, if this is not true throughout $I$, it will be true in the interval $x \geqq a^{\prime}$, where $a^{\prime}$ is sufficiently large. After establishing the existence of a continuous solution of (1) in this latter interval, we may by prolongement* extend it to all values of $x$ in $I$. 
It results from (13) and (14) that

$$
\int_{x}^{\infty}|K(x, t) f(t)| d t<|f(x)| \int_{x}^{\infty} \eta(t) d t<\theta|f(x)|
$$

Further, the integral

$$
\int_{x}^{\infty}|K(x, t) f(t)| d t
$$

converges uniformly in $I_{b}$, by the following (Weierstrass's) test. $\dagger$

Suppose that for all values of $x$ in the interval $a \leqq x \leqq b$ the function $F(x, t)$ satisfies the condition

$$
|F(x, t)|<M(t),
$$

where $M(t)$ is a positive function, independent of $x$. Then if the integral

converges, the integral

$$
\int_{x}^{\infty} M(t) d t
$$

$$
\int_{x}^{\infty} F(x, t) d t
$$

is absolutely and uniformly convergent for all values of $x$ in the interval $a \leqq x \leqq b$.

Let us write

$$
\int_{x}^{\infty}|K(x, t) f(t)| d t=\int_{x}^{b}|K(x, t) f(t)| d t+\int_{b}^{\infty}|K(x, t) f(t)| d t .
$$

The test applies at once to the integral last written if we take

$$
m=b, \quad F(x, t)=|K(x, t) f(t)|, \quad M(t)=N \eta(t),
$$

where $N$ is the maximum value of $|f(x)|$ in $I_{b}$.

Thus the conditions of Theorem 1 are satisfied in the present case, and by that theorem there exists a continuous solution of (1) of the form

If we put

$$
\phi(x)=f(x) \rho(x) \text {. }
$$

it appears by (4) that

$$
\rho(x)=1+\epsilon(x),
$$

and by (7) we have

$$
f(x) \epsilon(x)=\sum_{n=1}^{\infty} u_{n}(x),
$$

$$
|f(x) \epsilon(x)| \leqq \sum_{n=1}^{\infty}\left|u_{n}(x)\right|<\frac{\theta}{1-\theta}|f(x)|,
$$

or

$$
|\epsilon(x)|<\theta /(1-\theta) \text {. }
$$

† Cf., for instance, Bromwich, Infinite Series, p. 434. 
Now $\theta$ may be taken arbitrarily small if $x$ be taken sufficiently large, so that

This completes the proof.

$$
\lim _{x=\infty} \epsilon(x)=0 \text {. }
$$

5. Theorem 4. If there exist functions $h(x)$ and $\eta(x)^{*}$ such that the inequalities

$$
\begin{aligned}
& |K(x, t) f(t)|<h(x) \eta(t), \\
& |K(x, t)| h(t)<h(x) \eta(t)
\end{aligned}
$$

are satisfied in $T$, then equation (1) has in I a continuous solution $\phi(x)$ expressible in the form

$$
\phi(x)=f(x)+h(x) \epsilon(x),
$$

where $\epsilon(x)$ is continuous and has the property that

$$
\lim _{x=\infty} \epsilon(x)=0 \text {. }
$$

CoRollary. If there exists a function $\eta(x)$ such that the inequalities

$$
|K(x, t) f(t)|<\eta(t), \quad|K(x, t)|<\eta(t)
$$

are satisfied in $T$, then equation (1) has in I a continuous solution $\phi(x)$ expressible in the form

where

$$
\phi(x)=f(x)+\epsilon(x),
$$

$$
\lim _{x=\infty} \epsilon(x)=0 \text {. }
$$

From (15) and (16) it appears that (9) and (10) are satisfied. The integrals

$$
\int_{x}^{\infty}|K(x, t) f(t)| d t \text { and } \int_{x}^{\infty}|K(x, t)| h(t) d t
$$

are readily seen to satisfy the other conditions of Theorem 2 . Hence equation (1) has a continuous solution

where

$$
\phi(x)=f(x)+h(x) \epsilon(x),
$$

Now by (15)

$$
\epsilon(x)=\rho(x)=\frac{u_{1}(x)}{h(x)}+\frac{1}{h(x)} \sum_{n=2}^{\infty} u_{n}(x) .
$$

and by (12)

$$
\frac{\left|u_{1}(x)\right|}{h(x)}<\theta
$$

$$
\frac{1}{h(x)} \sum_{n=2}^{\infty}\left|u_{n}(x)\right|<\frac{\theta}{1-\theta},
$$

* The functions $h(x)$ and $\eta(x)$ have the same properties as in Theorem 2 and Theorem 3 respectively. 
so that

$$
|\epsilon(x)|<\theta+\theta /(1-\theta) .
$$

The desired result follows as in $\S 4$.

To prove the corollary, take $h(x)=1$.

6. Application. The foregoing results may be used in a variety of ways to discuss particular equations of the form (1). The following may be mentioned as a typical application:

Let $p(x), q(x)$, and $r(x)$ be polynomials in $x$ such that for large values of $x$

$$
R[p(x)]<R[q(x)]<R[r(x)],
$$

where $R[x]$ means the real part of $x$. If for sufficiently large values of $x$, and for values of $t \geqq x$, we may write, in Poincaré's sense,*

$$
\begin{gathered}
f(x) \sim e^{q(x)} x^{a}\left(a_{0}+\frac{a_{1}}{x}+\cdots\right), \\
K(x, t) \sim e^{p(x)} x^{\beta} e^{-r(t)} t^{\prime}\left(b_{0,0}+\frac{b_{1,0}}{x}+\frac{b_{0,1}}{t}+\frac{b_{2,0}}{x^{2}}+\cdots\right),
\end{gathered}
$$

where $\alpha, \beta, \gamma, a_{0}, \cdots, b_{0,0}, \cdots$ are determinate constants, then for the same values of $x$ the equation (1) has a continuous solution $\phi(x)$ such that we may write

$$
\phi(x) \sim f(x) .
$$

To prove this statement, we note first that $x$ may be taken so large that

$$
|K(x, t)|<N_{1}\left|e^{p(x)} x^{\beta}\right|\left|e^{-r(t)} t^{\gamma}\right|, \quad|f(x)|<N_{2}\left|e^{q(x)} x^{a}\right|,
$$

where $N_{1}$ and $N_{2}$ are certain constants. Thus

$$
|K(x, t) f(t)|<N_{1} N_{2}\left|e^{p(x)} x^{\beta} e^{q(t)-r(t)} t^{a+\gamma}\right| .
$$

Let us now apply Theorem 4, taking

$$
h(x)=\left|e^{p(x)} x^{\beta}\right|, \quad \eta(x)=N\left|e^{q(x) \rightarrow(x)}\right| x^{k},
$$

where $N$ is the larger of the quantities $N_{1}$ and $N_{1} N_{2}$, and $k$ is the larger of the quantities

Since

$$
R[\alpha+\beta], \quad R[\alpha+\gamma]
$$

the integral

$$
R[q(x)]<R[r(x)],
$$

$$
\int_{x}^{\infty} \eta(t) d t
$$

*Acta Mathematica, vol. 8 (1886), p. 296. 
exists. We have directly, by (17), the inequality

Also

$$
|K(x, t) f(t)|<h(x) \eta(t) .
$$

since

$$
|K(x, t)| h(t)<N_{1}\left|e^{p(x)} x^{\beta} e^{p(t)-r(t)} t^{\beta+\gamma}\right|<h(x) \eta(t)
$$

Thus the hypotheses of Theorem 4 are satisfied, and equation (1) has a continuous solution of the form

$$
\begin{aligned}
\phi(x) & =f(x)+h(x) \epsilon(x) \\
& =e^{q(x)} x^{a}\left[a_{0}+\frac{a_{1}}{x}+\cdots+\frac{a_{n}+\omega_{n}(x)}{x^{n}}\right]+\left|e^{p(x)} x^{e}\right| \epsilon(x),
\end{aligned}
$$

where $n$ is an arbitrary positive integer and

Put

$$
\lim _{x=\infty} \omega_{n}(x)=0, \quad \lim _{x=\infty} \epsilon(x)=0 .
$$

Then we have

$$
\begin{gathered}
\bar{\epsilon}(x)=\frac{\epsilon(x)\left|e^{p(x)} x^{\beta}\right|}{e^{p(x)} x^{\beta}} \\
\bar{\omega}_{n}(x)=\omega_{n}(x)+e^{p(x)-q(x)} x^{\beta-a+n} \bar{\epsilon}(x) .
\end{gathered}
$$

$$
\phi(x)=e^{q(x)} x^{a}\left[a_{0}+\frac{a_{1}}{x}+\cdots+\frac{a_{n}+\bar{\omega}_{n}(x)}{x^{n}}\right] .
$$

It appears by (18) that

$$
\lim _{x=\infty} \bar{\omega}_{n}(x)=0
$$

whence we may write

$$
\phi(x) \sim e^{q(x)} x^{a}\left(a_{0}+\frac{a_{1}}{x}+\cdots\right) .
$$

Thus $\phi(x)$ has an asymptotic expansion coinciding with that of $f(x)$, which was to be proved.

It is believed that the present theorems may prove useful in the study of certain problems in the theory of linear differential equations. (The writer hopes to consider applications of this type in a later paper.)

UNIVERSITY OF MichigaN. 